\title{
BMJ Open Use of implementation science in tobacco control intervention studies in the USA between 2000 and 2020: a scoping review protocol
}

\author{
Rebecca Selove (D) , ${ }^{1}$ Sarah Neil-Sztramko (D) , ${ }^{2}$ Jennifer Leng (D) , ${ }^{3}$ \\ Philip D Walker (1) , ${ }^{4}$ Ramzi George Salloum (1) ," Tamar Ginossar (D) ,6 \\ Carolyn Heckman (D) , ${ }^{7}$ Taneisha S Scheuermann (D) , ${ }^{8}$ Todd Combs (D) , 9 \\ Raquel Qualls-Hampton (D) , ${ }^{10}$ Rebecca Armstrong (i) , ${ }^{11}$ Shellie Ellis (i) ${ }^{8}$
}

To cite: Selove R, NeilSztramko S, Leng J, et al. Use of implementation science in tobacco control intervention studies in the USA between 2000 and 2020: a scoping review protocol. BMJ Open 2020;10:e038617. doi:10.1136/ bmjopen-2020-038617

- Prepublication history and additional material for this paper is available online. To view these files, please visit the journal online (http://dx.doi.org/10. 1136/bmjopen-2020-038617).

Received 17 March 2020 Revised 24 August 2020 Accepted 29 September 2020

Check for updates

(C) Author(s) (or their employer(s)) 2020. Re-use permitted under CC BY-NC. No commercial re-use. See rights and permissions. Published by BMJ.

For numbered affiliations see end of article.

Correspondence to Dr Rebecca Selove; rselove@tnstate.edu

\section{Abstract}

Introduction Despite continuing efforts to reduce tobacco use in the USA, decline in smoking rates have stalled and smoking remains a major contributor to preventable death. Implementation science could potentially improve uptake and impact of evidence-based tobacco control interventions; however, no previous studies have systematically examined how implementation science has been used in this field. Our scoping review will describe the use of implementation science in tobacco control in the USA, identify relevant gaps in research and suggest future directions for implementation science application to tobacco control.

Methods and analysis Our team, including a medical research librarian, will conduct a scoping review guided primarily by Arksey and 0'Malley's methodology. We will search English language peer-reviewed literature published from 2000 to 31 December 2020 for terms synonymous with 'tobacco use', 'prevention', 'cessation' and 'implementation science'. The databases included in this search are MEDLINE (PubMed), Embase (Ovid), CINAHL (EBSCOhost), PsycINFO (ProQuest), ERIC (ProQuest) and the Cochrane Library (Wiley). We will include cohort and quasi-experimental studies, single-group experiments and randomised trials that report qualitative and/or quantitative data related to applying implementation science to the planning and/or delivery of interventions to prevent or decrease the use of tobacco products. Studies must target potential or active tobacco users, intervention providers such as educators or healthcare professionals, or US policy-makers. A minimum of two reviewers will independently examine each title and abstract for relevance, and each eligible full text for inclusion and analysis. Use of implementation science, demonstrated by explicit reference to implementation frameworks, strategies or outcomes, will be extracted from included studies and summarised.

Ethics and dissemination This study is exempt from ethics board approval. We will document the equityorientation of included studies with the Preferred Reporting Items for Systematic Reviews and Meta-AnalysesEquity Extension checklist. Results will be submitted for conferences and peer-reviewed journals.
Strengths and limitations of this study

This scoping review protocol describes the approach to an investigation of the explicit use of implementation science in planning and/or delivering tobacco control interventions in the USA to reduce the prevalence of preventable diseases and deaths.

- This proposed review focuses on databases that are widely used by investigators who could benefit from learning about the application of implementation science in tobacco control research programmes.

- The study is designed to capture a comprehensive range of tobacco control programmes.

- The reviewers developed a logic model depicting the intersection of tobacco control interventions and implementation science to support the relevance of this study for improving population health and reducing tobacco-related health disparities.

- A limitation of the proposed study is that it is restricted to interventions in the USA. Studies that may meet inclusion criteria except for this element will be identified and considered for a later review.

Trial registration number Open Science Framework Registry (6YRK8).

\section{INTRODUCTION}

Tobacco use is the leading preventable cause of mortality in the USA and is associated with a wide variety of poor health outcomes and health disparities. ${ }^{1}$ Over the past 50 years, researchers and funding agencies have focused on developing and disseminating evidence-based programmes to prevent and reduce tobacco use and exposure to tobacco smoke. ${ }^{2}$ Community-based programmes that coordinated with state and national policies have been identified as most effective for achieving public health goals of reducing tobacco use, ${ }^{2}$ and clinical practice guidelines 
have been developed to treat tobacco dependence among current tobacco users. $^{3}$

Guidance regarding best practices for reducing tobacco use globally and in the USA has been available for decades. The WHO's Framework Convention on Tobacco Control, ${ }^{4}$ and the related MPOWER website, ${ }^{5}$ focus on national policies, including steps that reduce demand for tobacco products and exposure to second-hand smoke, and support cessation. Best practices for addressing tobacco use, provided by Centers for Disease Control and Protection (CDC) ${ }^{2}$ include goals and strategies that are similar to those of the WHO. Similarly, an Institute of Medicine committee report related to smoking cessation in the military ${ }^{6}$ outlined best practices that included establishing tobacco-free spaces and supporting tobacco use cessation.

Although tobacco use in the USA has declined since the first surgeon general's report linking it to lung cancer and other diseases in $1964,{ }^{1}$ the decrease in cigarette use plateaued early in the last decade. ${ }^{7}$ Recent trends show that tobacco product use, including nicotine delivery via cigarette alternatives, is on the rise. ${ }^{8}$ Furthermore, observed declines in tobacco use have occurred disproportionately among populations with more education, better health status, skilled jobs and higher household incomes, increasing disparities in health outcomes. ${ }^{9}$

Lags in effective translation of evidence to practice are common across health-related conditions, and can be addressed by applying best practices in implementation science. ${ }^{10}$ Implementation science is the use of scientific methods in studying the uptake and integration of evidence-based interventions into routine practice in nonresearch environments to improve the quality and benefit of those interventions. ${ }^{11} 12$ This field examines facilitators and barriers to establishing and sustaining evidencebased programmes in particular contexts to achieve specific implementation outcomes. ${ }^{13}$ Implementation science offers enhanced understanding of ways implementation strategies (such as developing a formal plan for implementing an intervention, or providing ongoing consultation to those who deliver the intervention ${ }^{14}$ ) that can be tested and successfully applied in varied contexts to maximise successful intervention outcomes. ${ }^{15}$

Implementation science resources include, for example, theoretically informed frameworks and models, ${ }^{16}{ }^{17}$ implementation strategies ${ }^{14}$ and measures of implementation processes and outcomes. ${ }^{18} 19$ The use of implementation science to enhance the impact of tobacco control programmes and policies has been identified as a priority for promoting prevention of tobacco use and cessation in the US population, especially among socioeconomically disadvantaged tobacco users. ${ }^{2021}$

Although implementation science is a young field, it has been applied across the cancer continuum. ${ }^{22}$ However, despite prioritisation of applying implementation science to improve public health, a review of ways in which implementation science has been used to plan and deliver tobacco control programmes and policies has not been previously published. Rosen et $^{2} \mathrm{l}^{23}$ examined 46 systematic reviews of tobacco control-related interventions and noted that variability in implementation quality limits reviewers' ability to interpret intervention effectiveness. A systematic review was conducted of studies that reported implementation strategies for a range of chronic disease prevention interventions, with control groups, and among the three studies that met their inclusion criteria, none focused on tobacco-related interventions. ${ }^{24}$ A recent scoping review ${ }^{25}$ described targeted populations and settings for tobacco control interventions, and found cessation interventions were the most common topics of systematic reviews. The authors suggested that the focus on cessation reflects an incomplete approach to tobacco control as recommended by the WHO. ${ }^{4}$ The authors indicated they plan to publish their observations regarding implementation challenges associated with cessation interventions.

A review of studies on smoking cessation interventions noted a failure to increase rates of tobacco cessation despite advances in pharmacotherapy and programmes demonstrated to be effective in research settings. ${ }^{26}$ The authors noted a lack of conclusive research as to whether this is due to insufficient reach of effective interventions, reduced effectiveness when programmes are translated to community settings or populations of community smokers for whom available interventions are less effective. Surgeon General, David Satcher, described many effective interventions that have been developed for advancing tobacco control and said, 'The challenge to public health professionals, healthcare systems and other partners in our national prevention effort is to implement these proven approaches' (U.S. Department of Health and Human Services, ${ }^{1}$ p12). This scoping review was undertaken to identify explicit use of implementation science across a comprehensive range of interventions as described by the $\mathrm{WHO}$ and the $\mathrm{CDC}^{2}$ to gauge the awareness of this field's potential contributions for improving effective use of tobacco control interventions.

In our preliminary search for published reports of the use of implementation science in planning and delivering tobacco control interventions, we considered projects described in PROSPERO, the Joanna Briggs Institute (JBI) Database of Systematic Reviews and Implementation Reports, and scoping reviews registered in the Center for Open Science. We found no existing or ongoing scoping reviews on the use of implementation science in tobacco control intervention research. The initial search by our medical librarian team member identified approximately 4500 titles, and we noted that a significant number of studies were conducted completely outside of the USA. Studies that describe the explicit use of implementation science tools appeared rarely in the initial samples of articles we reviewed. We opted to consider studies across a comprehensive range of tobacco use interventions because the focus of our review is on the application of implementation science rather than specific types of interventions or goals. Our initial screening suggests 


\begin{tabular}{|c|c|c|c|c|c|}
\hline \multirow[b]{2}{*}{$\begin{array}{l}\text { PROBLEMS: } \\
\text { Impact of efforts to } \\
\text { prevent and reduce } \\
\text { tobacco use and } \\
\text { exposure to } \\
\text { tobacco smoke in } \\
\text { the U.S. has } \\
\text { stalled; } \\
\text { tobacco use } \\
\text { disproportionately } \\
\text { affects certain } \\
\text { population groups. }\end{array}$} & \multirow[b]{2}{*}{\begin{tabular}{|l|}
\multicolumn{1}{c}{ INPUTS } \\
$\begin{array}{l}\text { Recommendations for } \\
\text { addressing problem: }\end{array}$ \\
1. Use evidence-based \\
interventions to promote a \\
tobacco-free culture, prevent \\
tobacco use initiation, \\
eliminate exposure to \\
second-hand smoke. \\
increase tobacco cessation, \\
and eliminate disparities in \\
tobacco use treatment.
\end{tabular}} & \multicolumn{2}{|c|}{ ACTIVITIES (examples) } & \multirow{2}{*}{ 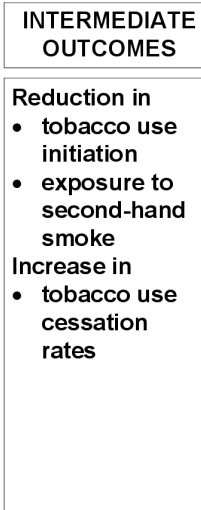 } & \multirow{2}{*}{ 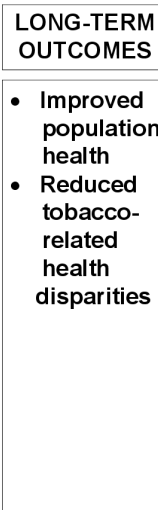 } \\
\hline & & $\begin{array}{l}\text { Public health interventions: } \\
\text { - Adopt policies to reduce tobacco } \\
\text { product demand, such as increasing } \\
\text { tobacco product tax, restricting } \\
\text { tobacco product marketing and } \\
\text { placement in retail settings, use in } \\
\text { public and work settings. } \\
\text { - Provide state cessation QuitLines. } \\
\text { - Delivering mass media messages } \\
\text { about harms of tobacco use. } \\
\text { - Train physicians, dentists, nurses to } \\
\text { assess tobacco use, deliver smoke- } \\
\text { reduction/ cessation/ second-hand } \\
\text { smoke reduction guidance and } \\
\text { support. }\end{array}$ & $\begin{array}{l}\text { Individual interventions: } \\
\text { - Offer tailored apps, } \\
\text { web-based cessation } \\
\text { services. } \\
\text { - Provide support and } \\
\text { FDA-approved } \\
\text { medication. } \\
\text { - Offer cessation } \\
\text { programs designed for } \\
\text { specific populations: } \\
\text { homeless people, } \\
\text { cancer survivors, } \\
\text { ethnic minorities, } \\
\text { pregnant women, } \\
\text { teenagers. }\end{array}$ & & \\
\hline & $\begin{array}{l}\text { 2. Use implementation } \\
\text { science to improve reach, } \\
\text { effectiveness, adoption, } \\
\text { implementation, and } \\
\text { maintenance of evidence- } \\
\text { based interventions. }\end{array}$ & \multicolumn{4}{|c|}{$\begin{array}{l}\text { Implementation science tools: Documented use of one or more of the following: (examples) } \\
\text { 1. Use of implementation science/knowledge translation models and frameworks } \\
\text { 2. Assessment of contextual factors in planning implementation of intervention } \\
\text { 3. Description of specific strategies used for implementation } \\
\text { 3. Measurement of implementation progress } \\
\text { 4. Assessment of implementation outcomes } \\
\text { 5. Assessment of implementation drivers }\end{array}$} \\
\hline
\end{tabular}

Figure 1 Logic model underlying tobacco control programme implementation scoping review.

that the number of studies that will qualify for inclusion will be manageable for data extraction and meaningful synthesis of the findings.

Describing the ways in which implementation science has been used in tobacco control interventions is essential to gain an understanding of the state of the field regarding the use of frameworks, models and strategies that can further reduce tobacco use rates and inequities. Thus, our goal is to examine peer-reviewed, published reports of tobacco control interventions in the USA to identify the use of implementation science in planning and/or delivering these interventions from 2000 through 31 December 2020. We developed a logic model to depict the rationale for this project, following the recommendations of Anderson $e t a l^{27}$ (figure 1).

This study will describe the nature of the use of implementation science frameworks and models, implementation strategies and measurement of implementation outcomes in research efforts to prevent tobacco use and second-hand smoke exposure and/or to promote smoking cessation. Results from this scoping review can be used to inform a research agenda for addressing gaps in, and advancing the application of implementation science in tobacco control to achieve greater impact, especially in addressing tobacco-related health disparities. ${ }^{28}$

\section{Review questions}

The primary research question for this scoping review is: How has implementation science been used in planning and delivering tobacco control interventions in the USA from 2000 to 31 December 2020? Our focus will be on ways researchers investigated the use of implementation science to plan and deliver tobacco control interventions. The inclusion criteria listed in table 1 describe specific elements of implementation science that will qualify studies to be examined in this review. The study is designed to address the following questions:
- What aspects of implementation science (such as use of implementation science frameworks and models, implementation strategies, and measurement of implementation processes and outcomes) appear explicitly in reports of tobacco control intervention studies?

- What types of interventions (ie, public health interventions such as classroom-based prevention education, tobacco use policies and electronic prompts for providers, as well as programmes that target individuals and families such as group counselling and text messages to support smoking cessation) are associated with explicit use of implementation science?

- What intervention goals (as described in table 2) are associated with the use of implementation science?

- Given the kinds of interventions that the proposed study identifies that have been planned and delivered with the benefit of implementation science, where are there gaps in implementation research? Specifically, in considering the dimensions of comprehensive tobacco control offered by the $\mathrm{WHO}^{4}$ and the $\mathrm{CDC},{ }^{2}$ are there kinds of interventions that less frequently used implementation science tools in planning and/ or delivery, where less is known about how implementation factors affected behavioural and clinical outcomes?

- Have the number of peer-reviewed published studies explicitly using implementation science changed over the past 19 years?

\section{METHODS}

The process for this scoping review will follow the guidance provided in Arksey and O'Malley's ${ }^{29}$ seminal paper, as well as the $\mathrm{JBI}^{3031}$ and other expert recommendations. ${ }^{32}$ The steps are: (1) identify a research question; (2) identify relevant published studies; (3) select studies that will be included in the scoping review, using clearly 
Table 1 Criteria for review of full texts for inclusion in study

\section{Inclusion}

- Article was published between 2000 and 31 December 2020.

- Study was conducted inside of USA (may include other countries as well, as long as USA is named also).

- The implementation of a tobacco control intervention or programme was studied.

1. Implementation science was explicitly used. The authors:described planned actions to promote human behaviour change in order to integrate tobacco control interventions into educational, community or clinical settings,

2. considered organisational constraints and facilitators that could affect uptake and delivery of the intervention, and

3. collected data regarding the processes and/or outcomes of their planned actions.

4. Data related to implementation science questions were collected and analysed.

Explicit use is further defined as reference to use of implementation science, knowledge translation or transfer, a specific implementation science framework or model, implementation strategies, assessment of implementation stages or implementation outcomes. Other elements may be included if they emerge in the course of the review.

\section{Exclusion}

- Completely outside of the USA

- Dissertation or thesis

- Essay or opinion piece

- Study protocol only

- Only describes guidelines

- Report of a conference presentation

Book

- Does not describe implementation of a tobacco control intervention

- Analysis of secondary surveillance or cross-sectional data by authors not involved in delivering intervention

- No indication that implementation science elements were used articulated inclusion and exclusion criteria; (4) extract data from each study to address the research questions and (5) summarise and disseminate the results of the data extraction and review process.

\section{Patient and public involvement}

No patient involvement was obtained in designing this scoping review protocol.

\section{Search strategy}

In consultation with our team's medical research librarian, and following JBI guidelines, a three-step search strategy will be used. ${ }^{30}$ The first step, an initial pilot search, was performed in MEDLINE (PubMed) in 2017. Second, additional search terms were added on review of the initial results and input from subject experts. The databases to be included in this search are MEDLINE (PubMed), Embase (Ovid), CINAHL (EBSCOhost), PsycINFO (ProQuest), ERIC (ProQuest) and the Cochrane Library (Wiley). All searches are limited to English language and publication dates from 1 January 2000 to 31 December 2020. The MEDLINE (PubMed) search strategy is defined in box 1 . Full details for the search strategy are provided in the online supplemental appendix 1.

\section{Study selection}

All identified references will be uploaded into Covidence systematic review software (Veritas Health Innovation, Melbourne, Australia). Duplicate citations will be removed using the Covidence software. Each title and abstract will be reviewed for relevance by two of the study authors. At the title and abstract screening phase, studies that will be included must describe a tobacco control intervention conducted in the USA, and be published between 2000 and 31 December 2020. Conflicts regarding inclusion for full-text review will be resolved by the senior author (RS) and through team discussion where necessary.

During the initial title and abstract screen, relevant systematic reviews will be identified for hand searching. For titles found through hand searches of systematic reviews that are not already in the original data set, the associated abstracts will be reviewed by two of the study authors using the same inclusion and exclusion criteria given in table 1. Full texts will be retrieved for final eligibility screening using the inclusion and exclusion criteria presented below. Each full text will be reviewed for inclusion in the final study by two members of the study team, with conflicts resolved through team discussion. During full-text reviews, members of the study team will hand search for citations of related publications that might provide more complete descriptions of the tobacco control intervention. Titles and abstracts of publication identified by hand searches will be reviewed by two members of the study team as described above.

\section{Inclusion criteria}

We will use the population-concept-context framework described by the scoping review guidelines of the $\mathrm{JBI}^{30}$ as one dimension of our inclusion criteria. Based on the variety of tobacco control interventions, we expect that populations represented in included studies will vary. Tobacco control interventions are designed to prevent use, as well as reduce primary and secondary exposure to tobacco. Thus, study populations can include nonsmokers, combustible tobacco and smokeless tobacco 
Table 2 Tobacco control programmes/interventions: goals, target audience and components

Programme goals

Programme/intervention components

\begin{tabular}{|c|c|c|c|c|}
\hline ure & & $\begin{array}{l}\text { Eliminate } \\
\text { second-hand } \\
\text { smoke exposure }\end{array}$ & $\begin{array}{l}\text { Ise } \\
\text { co } \\
\text { tion }\end{array}$ & $\begin{array}{l}\text { in } \\
\text { tr }\end{array}$ \\
\hline
\end{tabular}

at two levels

free culture initiation smoke exposure cessation treatment

Public health interventions: society (government and industry); community (eg, healthcare providers, schools and educators, housing complexes and workplaces/retailers)

$\begin{array}{lllllll}\text { Policy interventions } & \mathrm{PH} 1 & \mathrm{PH} 2 & \mathrm{PH} 3 & \mathrm{PH} 4 & \mathrm{PH} 5\end{array}$

- Tobacco use restrictions such as bans in restaurants, work places, parks and cars with child passengers

- Multiuse housing bans

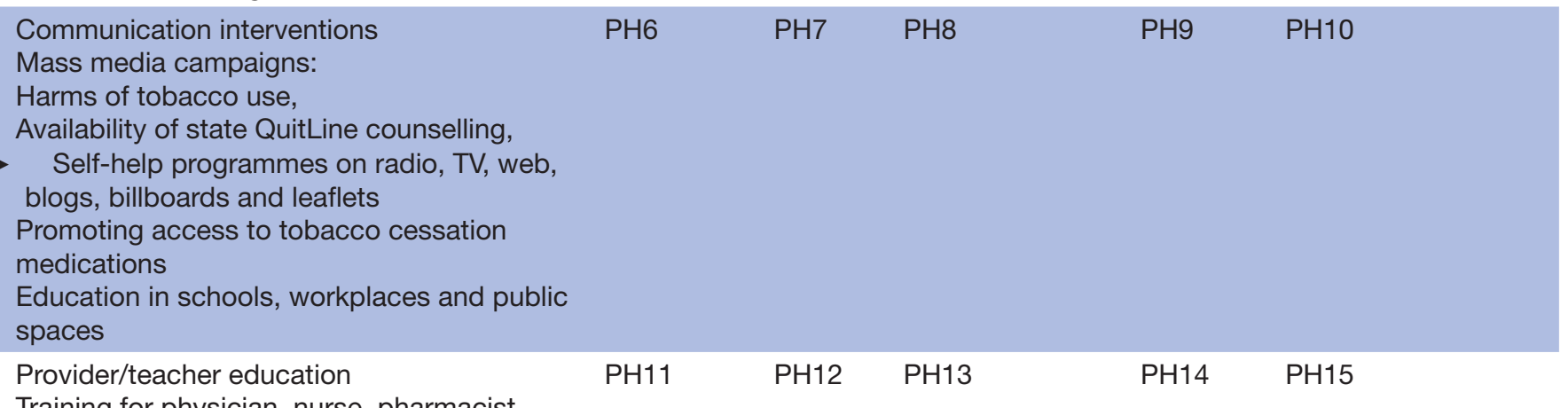

Training for physician, nurse, pharmacist,

dentist and teacher

Electronic/written prompts to check tobacco use status

\begin{tabular}{|c|c|c|c|c|c|}
\hline $\begin{array}{l}\text { Tobacco screening/other intervention } \\
\text { guideline }\end{array}$ & PH16 & $\mathrm{PH} 17$ & PH18 & PH19 & $\mathrm{PH} 20$ \\
\hline
\end{tabular}

Individual interventions: family, individual adults, children and youth

\begin{tabular}{|c|c|c|c|c|c|}
\hline $\begin{array}{l}\text { Communication interventions } \\
\text { Text messages for quitting } \\
\text { Web-based media literacy education }\end{array}$ & 121 & 122 & 123 & 124 & 125 \\
\hline $\begin{array}{l}\text { Behavioural therapies and medication } \\
\text { Brief advice from healthcare provider-3 or } 5 \\
\text { A's } \\
\text { Provider referral to QuitLine } \\
\text { Multisession QuitLine counselling } \\
\text { Face-to-face group and individual counselling } \\
\text { Food and drug administration-approved } \\
\text { medications and NRT } \\
\text { App and web-based programmes }\end{array}$ & 126 & 127 & 128 & 129 & 130 \\
\hline $\begin{array}{l}\text { Cessation programmes for special populations I } \\
\text { Homeless people, smokers with mental health } \\
\text { and/or substance use disorders, cancer } \\
\text { survivors, ethnic minorities and pregnant } \\
\text { women }\end{array}$ & 131 & I32 & 133 & 134 & 135 \\
\hline
\end{tabular}

NRT, nicotine replacement therapy.

users, and individuals exposed to second-hand smoke. These populations include persons of all races and ethnicities, and range in age from infants to older adults. ${ }^{9} 33$ Targets of tobacco control interventions include pregnant women, school children, parents, healthcare providers, smoking cessation counsellors, teachers, public health workers, policy makers, media stakeholders and proprietors of establishments that sell tobacco products or regulate exposure to tobacco smoke. With regard to contexts, tobacco control interventions are delivered in a wide variety of settings, including healthcare providers' offices, hospitals, classrooms, daycare centres, afterschool programmes, community centres, faith communities and more. Because of this diversity, we will not exclude studies based on participant characteristics or programme settings. 


\section{Box 1 Search strategy for MEDLINE (PubMed)}

("Tobacco Products"(MeSH) OR "Tobacco Use"(MeSH) OR "Tobacco Smoke Pollution" (MeSH) OR "Smoking"(MeSH) OR tobacco (tiab) OR tobacco use (tiab) OR smoking (tiab) OR second handsecondhand smoke exposure (tiab) OR second handsecondhand smoke (tiab) OR tobacco use initiation (tiab) OR smoking initiation (tiab) OR "Tobacco Use Cessation"(MeSH) OR tobacco control (tw) OR "smoking cessation" (MeSH Terms) OR smoking cessation (tw)) AND ("Smoking Prevention"(MeSH) OR smoking prevention (tw) OR "Health Promotion" (MeSH) OR health promotion (tw) OR "Health Education" (MeSH) OR health education (tw) OR programprogramme (tw) OR programsprogrammes (tw) OR intervention (tw) OR interventions (tw) OR 'Policy' (MeSH) OR "Smoke-Free Policy" (MeSH) OR "Social Control Policies" (MeSH) OR "Organizational Organisational Policy" (MeSH) OR "Public Policy" (MeSH) OR policy (tw) OR policies (tw) OR public policy (tw) OR health policy (tw)) AND (implementation science (tw) OR implementation(tw) OR "diffusion of innovation" (MeSH Terms) OR implementation frameworks (tw) OR implementation models (tw) OR implementation study (tw) OR translational research (tw) OR "translational medical research" (MeSH Terms) OR knowledge translation (tw)) AND (Clinical Trial (ptyp) OR Comparative Study (ptyp) OR Controlled Clinical Trial (ptyp) OR Evaluation Studies (ptyp) OR Observational Study (ptyp) OR RandomizedRandomised Controlled Trial (ptyp) OR "Qualitative Research" (MeSH) OR "Prospective Studies" (Mesh) OR "Cohort Studies" (MeSH) OR Meta-Analysis (ptyp) OR systematic (sb) OR evaluation studies (ptyp) OR evaluation studies (tw) OR clinical trial (tw) OR comparative study (tw) OR observational study (tw) OR qualitative research (tw) OR "programprogramme evaluation" (MeSH Terms) OR programprogramme evaluation (tw) OR hybrid design (tw) OR experimental (tw) OR mixed methods study (tw)) AND (“2000/01/01” (PDAT) : "2020/12/31" (PDAT)) AND English (lang)

This study will examine the intersection of two key concepts: tobacco control programmes and implementation science. For this study, tobacco control programmes or interventions are defined as activities that aim to achieve one or more of the following goals: (1) promote tobacco-free culture; (2) prevent initiation of tobacco use; (3) eliminate second-hand smoke exposure; (4) increase tobacco cessation; or (5) eliminate disparities in tobacco use treatment. ${ }^{4}{ }^{6} 34$ Examples of such interventions include, but are not limited to, public health interventions (eg, taxation, mass media campaigns, tobacco use restrictions or policies relevant to tobacco retail environments) or individual-level interventions (eg, healthcare provider training, tailored communication interventions, pharmacotherapy or structured counselling). ${ }^{2}$ We drew from the CDC's best practices for tobacco control, ${ }^{2}$ the WHO's Framework Convention on Tobacco Control ${ }^{4}$ and the related MPOWER website, ${ }^{5}$ and an Institute of Medicine committee report related to smoking cessation in the military, ${ }^{6}$ to develop a matrix of tobacco control interventions presented in table 2 . We will use this matrix to categorise reports of interventions to address Objective 3, and will compare our results to those of Halas et $a l^{25}$ who found that tobacco use cessation was the most common goal of studies they examined.
The inclusion criteria for use of implementation science were developed by reviewing seminal writings in the field, for example, ${ }^{10131417}$ and operational definitions reported in a scoping review of implementation science associated with nursing interventions in Germanspeaking countries. ${ }^{35}$ We also asked five leading scholars in the implementation science field how they would determine if a study should be included in this scoping review. This led to identification of three broad elements for determining that implementation science was used: investigators (1) described planned actions to promote human behaviour change in order to integrate evidencebased tobacco control interventions into educational, community or clinical settings, (2) considered organisational constraints and facilitators that could affect uptake and delivery of the intervention, and (3) collected data regarding the processes and/or outcomes of their planned actions.

To address research question 1 (What aspects of implementation science appear in the studies?), the multidisciplinary scoping review team reviewed a sample of articles that would be considered for the scoping review, and identified two categories of implementation science use: tier 1 and tier 2. Tier 1 studies include elements that are explicitly labelled as implementation science, such as: use of a specific implementation science framework such as the Interactive Systems Framework ${ }^{36}$ or an implementation toolkit ${ }^{37} 38$ for planning adoption of an intervention; use of specific implementation strategies ${ }^{1439}$ for enhancing delivery of an intervention; measuring stages of implementation ${ }^{40}$ during the process of delivering an intervention; or measurement of implementation outcomes as articulated by Proctor $e t a l^{13}$ as part of evaluating an intervention.

Implementation outcomes of interest include, but are not limited to, rates of intervention adoption, acceptability to patients, feasibility, appropriateness, costs, fidelity, penetration and sustainability; ${ }^{13}$ or broader service outcomes assessing processes of care such as safety, timeliness, efficiency, effectiveness, equity or patientcenteredness. ${ }^{13} 42$ Provider acceptability, self-efficacy for delivering an intervention as well as satisfaction with outcomes of an intervention will also be considered as aspects of implementation science, among variables that may be associated with implementation outcomes ${ }^{43}$ as listed previously. Multiple definitions and terminologies are used globally to convey the use of science to translate evidence-based research into practice, ${ }^{44}{ }^{45}$ for example, knowledge translation and translation of research into practice, and investigators may use these terms to refer to such planned activities. ${ }^{38} 46$

We anticipate that we will find peer-reviewed articles indicating that investigators assessed implementation facilitators and barriers, ${ }^{43}$ such as attitudes of key stakeholders toward a proposed intervention, organisational capacity for accommodating a new intervention, or community readiness to adopt and implement a tobaccorelated policy, without explicitly describing their work as 
implementation science. We will tag these tier 2 articles for a separate review.

This review will include studies published between 2000 and 31 December 2020. We chose the year 2000 as it represents the beginning of 'preparation' ${ }^{47}$ for application of the resources of the emerging field, and the early stage of an era when implementation research and implementation science began expanding as a focus in the peer-reviewed literature. ${ }^{48} 49$ We will limit our review to studies conducted in the USA because policies, laws, regulations (eg, on advertising) and cultural norms related to tobacco vary widely across countries and regions of the world. This inclusion criteria reduces the heterogeneity of contextual factors, which are prime considerations for implementation science, ${ }^{49}$ and enhances the feasibility of our undertaking. Studies conducted only outside the USA that may meet all other criteria for this scoping review will be identified for a future project.

Qualitative and quantitative empirical studies published in peer-reviewed journals will be eligible for inclusion. Study designs may include prospective cohort studies, natural experiments, quasi-experimental studies, singlegroup experiments and/or randomised controlled trials. Studies will be eligible if they report on primary data collection related to the process of implementing an intervention, whether or not they report evaluation of the effectiveness of the intervention. Dissertations, theses, reports of conference presentations, letters, guidelines, grey literature and books will be excluded, as we are limiting the review to publications that are more readily accessible to the broader scientific and practitioner community. As we are interested in articles in which use of implementation science is readily apparent to researchers and practitioners, we will not seek additional information from authors to investigate use of implementation science that is not reported in their published work.

\section{Assessment of methodological quality}

The purpose of this review is to identify how implementation science has been used across a wide range of study designs in tobacco control research. We do not plan to assess the methodological quality of the interventions themselves or provide a summary of what kind of interventions are effective. We plan to describe findings regarding the explicit application of implementation science including frameworks and models, strategies, assessment and measurement of implementation barriers and facilitators, stages and outcomes. Our critical appraisal will focus on uses and gaps of implementation science in included studies. ${ }^{30}$

\section{Extraction of results}

Once full texts to be included in the scoping review have been identified, two members of the study team will independently extract study characteristics from each one using a structured data extraction form in Covidence. Elements to be extracted include: (a) characteristics of the population targeted by the intervention, including sex, age, tobacco use status, ethnicities and roles (such as dentists or nurses, pregnant women and policy makers); (b) context in which the intervention is implemented, such as classroom, hospital, multiunit housing and county; (c) specific types and goals of interventions as categorised in table 2, as well as if and how the authors described the evidence-base for the intervention; (d) what aspects of implementation science were used in the design, delivery and/or evaluation of the intervention, such as specific implementation frameworks or models, implementation strategies, or measurement of intervention processes or outcomes; and (e) contributions of implementation science to the study if identified by the study's authors.

Discrepancies in extracted responses will be resolved through team discussion when necessary. The data extraction process will be trialled by the study team prior to execution to ensure consistency and relevance of fields before proceeding to full data extraction.

\section{Data synthesis}

Following data extraction, frequencies of study characteristics will be calculated where possible. In addition, the study team will conduct a narrative synthesis ${ }^{50}$ of characteristics of populations, content and contexts in included studies. The purpose of this analytic approach is to tell a story about use of implementation science in tobacco control research in the USA, including description of patterns that may emerge, such as target audiences, intervention goals or settings associated with implementation science use, as well as the impact of its use. The critical reflection required in this process has the potential for generating hypotheses ${ }^{50}$ regarding evolution of the use of implementation science in this field. A meta-analysis will not be conducted, as this will not be necessary to address the research question.

\section{Potential implications of findings}

Preliminary reviews of full text suggest that implementation science has not been used extensively in efforts to prevent and reduce tobacco use in the USA. We anticipate that the results of this scoping review will contribute to the knowledge base of implementation researchers in describing the extent and nature of implementation science application to public health and individual levels of tobacco control (table 2). This study will also provide investigators who implement tobacco control interventions across the range of goals described by the $\mathrm{CDC}$ and the $\mathrm{WHO},{ }^{4}$ with examples of studies that are informed by implementation science, as well as gaps in applications from this field. As the scoping review will include almost two decades of research, we anticipate that we will identify trends in implementation science use in tobacco control intervention research as the field has matured over time.

\section{Ethics and dissemination}

One goal of this study is to contribute to improvement in tobacco control interventions in reducing health disparities (figure 1). Efforts to promote effective tobacco control 
reflect commitments to social justice ${ }^{51}$ and implementation science can substantially improve the outcomes of these efforts. We will document the equity-orientation of included studies with the Preferred Reporting Items for Systematic Reviews and Meta-Analyses (PRISMA)-Equity Extension checklist. ${ }^{52}$

The findings from this study will be disseminated via peer-reviewed publications and conference presentations for audiences interested in tobacco control and implementation science. All results will be prepared in accordance with JBI guidelines ${ }^{30}$ and checklists for PRISMA, ${ }^{53}$ the Extension for Scoping Reviews (PRISMA-ScR) ${ }^{52}$ and equity reporting. ${ }^{54}$ A PRISMA flow diagram ${ }^{53}$ will indicate the numbers of articles identified in each search method, duplicates removed, and number of studies excluded and included, along with reasons for exclusion at the full-text review level. The main findings will be presented using tables and a narrative description that will detail the results in view of the objectives and research questions of the scoping review. A list of the included studies, along with their key characteristics, will be provided in the primary manuscript reporting the results of this review.

\section{Author affiliations}

${ }^{1}$ Center for Prevention Research, Tennessee State University, Nashville, Tennessee, USA

${ }^{2}$ Faculty of Health Sciences, McMaster University, Hamilton, Ontario, Canada ${ }^{3}$ Immigrant Health and Cancer Disparities Center, Memorial Sloan Kettering Cancer Center, New York, NY, USA

${ }^{4}$ Eskind Biomedical Library, Vanderbilt University, Nashville, Tennessee, USA ${ }^{5}$ Health Outcomes \& Biomedical Informatics, University of Florida College of Medicine, Gainesville, Florida, USA

${ }^{6}$ Communications \& Journalism, University of New Mexico, Albuquerque, New Mexico, USA

${ }^{7}$ Division of Medicine, Rutgers Cancer Institute of New Jersey, New Brunswick, New Jersey, USA

${ }^{8}$ Population Health, University of Kansas School of Medicine, Lawrence, Kansas, USA

${ }^{9}$ Center for Public Health Systems Science, Washington University in Saint Louis,

Saint Louis, Missouri, USA

${ }^{10}$ Meharry Medical College, Nashville, Tennessee, USA

${ }^{11}$ Australian Institute of Family Studies, Melbourne, Victoria, Australia

Acknowledgements The authors would like to thank the following individuals who were consulted regarding the definition of use of implementation science developed for this paper: Sarah Birken, Ross Brownson, Anne Sales and Michel Wensing.

Contributorship statement All co-authors have participated via email, phone, or in-person in the development of the protocol for this project. RS conceptualised the initial project, coordinated the research team in developing the protocol, was lead author for the protocol manuscript, participated in writing, reviewing and editing the manuscript, and drafted the detailed response to reviewers. SN-S contributed to developing the protocol and protocol manuscript, and participated in writing, reviewing and editing the manuscript, provided consultation regarding the Covidence software used for data review, and provided suggestions regarding scoping review procedures. JL contributed to developing the protocol, writing, reviewing and editing the manuscript. PDW, an experienced medical research librarian, developed and conducted the literature search, writing, reviewing and editing the manuscript. RGS contributed to developing the protocol, writing, reviewing and editing the manuscript. TG contributed to developing the protocol, and writing, reviewing and editing the manuscript. $\mathrm{CH}$ contributed to developing the protocol, and writing, reviewing and editing the manuscript. TSS contributed to developing the protocol, writing, reviewing and editing the manuscript, and provided suggestions regarding scoping review procedures. TC contributed to developing the protocol, writing, reviewing and editing the manuscript, and provided suggestions regarding scoping review procedures. $\mathrm{RQ}-\mathrm{H}$ contributed to developing table 2 and the protocol. RA contributed to developing the protocol and protocol manuscript. SE contributed to developing the protocol, developed the first draft of the protocol manuscript, reviewed and edited it, and contributed to editing the detailed description of responses to reviewers.

Funding Support for this project came in part from the National Institutes of Health Mentored Training for Dissemination and Implementation Research in Cancer Program (MT-DIRC) (grant number 5R25CA171994), the US Department of Veterans Affairs and the Cancer Research Network. RS is supported by the National Cancer Institute through the Meharry-Vanderbilt-Tennessee State University Cancer Partnership (U54CA163066). JL is supported by the National Cancer Institute: Core Cancer Center Support Grant (P30CA008748). SE is supported by the National Institute of General Medical Sciences COBRE grant (P20GM130423). TSS is funded by a NIH/NIDA K01 (K01 DA040745).

Competing interests None declared.

Patient consent for publication Not required.

Provenance and peer review Not commissioned; externally peer-reviewed.

Supplemental material This content has been supplied by the author(s). It has not been vetted by BMJ Publishing Group Limited (BMJ) and may not have been peer-reviewed. Any opinions or recommendations discussed are solely those of the author(s) and are not endorsed by BMJ. BMJ disclaims all liability and responsibility arising from any reliance placed on the content. Where the content includes any translated material, BMJ does not warrant the accuracy and reliability of the translations (including but not limited to local regulations, clinical guidelines, terminology, drug names and drug dosages), and is not responsible for any error and/or omissions arising from translation and adaptation or otherwise.

Open access This is an open access article distributed in accordance with the Creative Commons Attribution Non Commercial (CC BY-NC 4.0) license, which permits others to distribute, remix, adapt, build upon this work non-commercially, and license their derivative works on different terms, provided the original work is properly cited, appropriate credit is given, any changes made indicated, and the use is non-commercial. See: http://creativecommons.org/licenses/by-nc/4.0/.

\section{ORCID iDs}

Rebecca Selove http://orcid.org/0000-0003-2929-8763

Sarah Neil-Sztramko http://orcid.org/0000-0002-9600-3403

Jennifer Leng http://orcid.org/0000-0003-4925-2842

Philip D Walker http://orcid.org/0000-0002-1712-2886

Ramzi George Salloum http://orcid.org/0000-0002-8139-2418

Tamar Ginossar http://orcid.org/0000-0002-0136-6675

Carolyn Heckman http://orcid.org/0000-0003-1016-3965

Taneisha S Scheuermann http://orcid.org/0000-0001-9543-903X

Todd Combs http://orcid.org/0000-0003-1015-6589

Raquel Qualls-Hampton http://orcid.org/0000-0003-2401-4231

Rebecca Armstrong http://orcid.org/0000-0003-4146-7427

Shellie Ellis http://orcid.org/0000-0002-3599-0804

\section{REFERENCES}

1 U.S. Department of Health and Human Services. The health consequences of smoking- 50 years of progress. A report of the surgeon General. Atlanta, GA: U.S. Department of Health and Human Services, Centers for Disease Control and Prevention, National Center for Chronic Disease Prevention and Health Promotion, Office of Smoking and Health, 2014.

2 Centers for Disease Control and Prevention. Best practices for comprehensive tobacco control programs - 2014. Atlanta, GA: Department of Health and Human Services, National Center for Chronic Disease Prevention and Health Promotion, Office on Smoking and Health, 2014.

3 Fiore M, Jaen C, Baker T, et al. Treating tobacco use and dependence: 2008 update. Rockville, MD: Department of Health and Human Services, Public Health Service, 2008.

4 WHO. Who framework convention on tobacco control. Geneva: WHO, 2005.

5 World Health Organization. Tobacco free initiative (TFI). tobacco free initiative (TFI). Available: https://www.who.int/tobacco/mpower/ publications/en/

6 Institute of Medicine. Combating tobacco use in military and veteran populations. Washington, DC: National Academies Press, 2009.

7 Balogh E, Patlak M, Nass SJ. Reducing tobacco-related cancer incidence and mortality: workshop summary. Washington, DC: National academies press, 2013. 
8 Kasza KA, Ambrose BK, Conway KP, et al. Tobacco-product use by adults and youths in the United States in 2013 and 2014. N Engl J Med Overseas Ed 2017;376:342-53.

9 Wang TW, Asman K, Gentzke AS, et al. Tobacco product use among adults - United States, 2017. MMWR Morb Mortal Wkly Rep 2018;67:1225-32.

10 Brownson RC, Colditz GA, Proctor EK, eds. Dissemination and implementation research in health: translating science to practice. New York: Oxford University Press, 2012.

11 Eccles MP, Mittman BS. Welcome to implementation science. Implement Sci 2006;1:1748-5908.

12 Nilsen P, Birken SA. Handbook on implementation science. Cheltenham UK: Edward Elgar Publishing, 2020.

13 Proctor E, Silmere H, Raghavan R, et al. Outcomes for implementation research: conceptual distinctions, measurement challenges, and research agenda. Adm Policy Ment Health 2011;38:65-76.

14 Powell BJ, Waltz TJ, Chinman MJ, et al. A refined compilation of implementation strategies: results from the expert recommendations for implementing change (ERIC) project. Implement Sci 2015;10:21.

15 Chilenski SM, Greenberg MT, Feinberg ME. Community readiness as a multidimensional construct. J Community Psychol 2007;35:347-65.

16 Bauer MS, Damschroder L, Hagedorn H, et al. An introduction to implementation science for the non-specialist. BMC Psychol 2015;3:32.

17 Tabak RG, Khoong EC, Chambers D, et al. Models in dissemination and implementation research: useful tools in public health services and systems research. Front Public Health Serv Syst Res 2013;2:8

18 Gem: Grid-Enabled measures database. Available: https://www.gembeta.org/Public/Home.aspx

19 Huijg JM, Gebhardt WA, Dusseldorp E, et al. Measuring determinants of implementation behavior: psychometric properties of a questionnaire based on the theoretical domains framework. Implement Sci 2014:9:33.

20 U.S. Department of Health and Human Services. . limproving smoking cessation in socioeconomically disadvantaged populations via scalable interventions (R01), 2016. Available: https://grants.nih. gov/grants/guide/pa-files/par-16-202.html

21 U.S. Department of Health and Human Services. U.S. tobacco control policies to reduce health disparities (R01 clinical trial optional), 2018. Available: https://grants.nih.gov/grants/guide/pafiles/par-18-675.htm

22 Chambers D, Vinson C, Norton W, eds. Advancing the science of implementation across the cancer continuum. New York, NY: Oxford University Press, 2019

23 Rosen LJ, Ben Noach M, Rosenberg E. Missing the forest (plot) for the trees? A critique of the systematic review in tobacco control. BMC Med Res Methodol 2010;10:34.

24 McFadyen T, Chai LK, Wyse R, et al. Strategies to improve the implementation of policies, practices or programmes in sporting organisations targeting poor diet, physical inactivity, obesity, risky alcohol use or tobacco use: a systematic review. BMJ Open 2018;8:e019151.

25 Halas G, Schultz ASH, Rothney J, et al. A scoping review of foci, trends, and gaps in reviews of tobacco control research. Nicotine Tob Res 2020;22:599-612.

26 Zhu S-H, Lee M, Zhuang Y-L, et al. Interventions to increase smoking cessation at the population level: how much progress has been made in the last two decades? Tob Control 2012;21:110-8.

27 Anderson LM, Petticrew M, Rehfuess E, et al. Using logic models to capture complexity in systematic reviews: logic models in systematic reviews. Res Synth Methods 2011;2:33-42.

28 Chinman M, Woodward EN, Curran GM, et al. Harnessing implementation science to increase the impact of health equity research. Med Care 2017;55:S16-23.

29 Arksey H, O'Malley L. Scoping studies: towards a methodological framework. Int J Soc Res Methodol 2005;8:19-32.

30 Joanna Briggs Institute. Joanna Briggs Institute Reviewer's Manual. Adelaide, AU: The Joanna Briggs Institute, 2015.

31 Khalil H, Peters M, Godfrey CM, et al. An evidence-based approach to scoping reviews. Worldviews Evid Based Nurs 2016;13:118-23.

32 Daudt HML, van Mossel C, Scott SJ. Enhancing the scoping study methodology: a large, inter-professional team's experience with Arksey and O'Malley's framework. BMC Med Res Methodol 2013;13:48

33 Gentzke AS, Creamer M, Cullen KA, et al. Vital signs: tobacco product use among middle and high school students - United States, 2011-2018. MMWR Morb Mortal Wkly Rep 2019;68:157-64.
34 Best practices for comprehensive tobacco control programs: 2014. Tob Control 2014:144.

35 Hoben M, Berendonk C, Buscher I, et al. Scoping review of nursingrelated dissemination and implementation research in Germanspeaking countries: mapping the field/scoping review zum stand Der pflegebezogenen Disseminations- und Implementierungsforschung in deutschsprachigen Ländern: eine Bestandsaufnahme. Int $\mathrm{J}$ Health Prof 2014;1:34-49.

36 Wandersman A, Duffy J, Flaspohler P, et al. Bridging the gap between prevention research and practice: the interactive systems framework for dissemination and implementation. Am J Community Psychol 2008;41:171-81.

37 Washington University in St. Louis. Dissemination and implementation at Washington University in St. Louis. D \& I toolkits. Available: https://sites.wustl.edu/wudandi/di-toolkits/

38 Barac R, Stein S, Bruce B, et al. Scoping review of toolkits as a knowledge translation strategy in health. BMC Med Inform Decis Mak 2014;14:121.

39 Proctor EK, Powell BJ, McMillen JC. Implementation strategies: recommendations for specifying and reporting. Implement Sci 2013;8:139

40 Meyers DC, Durlak JA, Wandersman A. The quality implementation framework: a synthesis of critical steps in the implementation process. Am J Community Psychol 2012;50:462-80.

41 Saldana $\mathrm{L}$. The stages of implementation completion for evidencebased practice: protocol for a mixed methods study. Implementation Sci 2014;9:1.

42 Institute of Medicine (U.S), Committee on Quality of Health Care in America. Crossing the quality chasm: a new health system for the 21st century. Washington, DC: national Academy press, 2001 Available: http://public.eblib.com/choice/publicfullrecord.aspx? $p=$ 3375215

43 Damschroder LJ, Aron DC, Keith RE, et al. Fostering implementation of health services research findings into practice: a consolidated framework for advancing implementation science. Implement Sci 2009;4:50.

44 Rabin BA, Brownson RC. Developing the terminology for dissemination and implementation research. In: Brownson RC, Colditz GA, Proctor E, eds. Dissemination and implementation research in health: translating science into practice. New York, NY: Oxford university press, 2012: 23-51.

45 Sales A. Implementation science: how can it support healthcare research? training in implementation: actionable research approaches (TIARA). Kansas City, KA, 2019.

46 Slaughter SE, Zimmermann GL, Nuspl M, et al. Classification schemes for knowledge translation interventions: a practical resource for researchers. BMC Med Res Methodol 2017;17:161.

47 Chambers D. Forward. in: dissemination and implementation research in health: translating science into practice. New York: Oxford University Press, 2012: vii-x.

48 Dearing JW, Kee K. Historical roots of dissemination and implementation science. In: Brownson RC, Colditz GA, Proctor EK, eds. Dissemination and implementation research in health: translating science into practice. New York: Oxford University Press, 2012: 55-71.

49 Kerner JF, Glasgow RE, Vinson CA. A history of the National Cancer Institute's support for implementation science across the cancer control continuum: Context counts. In: Chambers D, Vinson CA, Norton W, eds. Advancing the science of implementation across the cancer continuum. New York, NY: Oxford University Press, 2019.

50 Tricco AC, Soobiah C, Antony J, et al. A scoping review identifies multiple emerging knowledge synthesis methods, but few studies operationalize the method. J Clin Epidemiol 2016;73:19-28.

51 Borges LC, Menezes HZde, Souza IMLde. Dilemas na implementação da convenção-quadro para o controle do tabaco da organização mundial da saúde. Cad. Saúde Pública 2020;36:e00136919.

52 Tricco AC, Lillie E, Zarin W, et al. PRISMA extension for scoping reviews (PRISMA-ScR): checklist and explanation. Ann Intern Med 2018:169:467.

53 Moher D, Liberati A, Tetzlaff J, et al. Preferred reporting items for systematic reviews and meta-analyses: the PRISMA statement. PLoS Med 2009;6:6.

54 Welch V, Petticrew M, Tugwell P, et al. PRISMA-Equity 2012 extension: reporting guidelines for systematic reviews with a focus on health equity. PLoS Med 2012;9:e1001333. 